\title{
Energy Aspects in Spray Formation by Homogenous Flash Boiling Process
}

\author{
Yahav Moshkovich*1, Yeshayahou Levy ${ }^{1}$, Ilai Sher ${ }^{2}$, Eran Sher ${ }^{1}$ \\ ${ }^{1}$ Faculty of Aerospace Engineering, Technion - Israel Institute of Technology, Haifa, Israel \\ ${ }^{2}$ Cranfield University, Cranfield, Bedfordshire MK43 OAL, United Kingdom \\ *Corresponding author: yahavmoshko@gmail.com
}

\begin{abstract}
When a pressurized bubbly mixture is driven out through an orifice, the mixture pressure abruptly drops and the bubbles undergo a rapid expansion process, which under some circumstances results in a rapid disintegration of the liquid bulk into small droplets (atomization). Depending on the initial conditions, heterogeneous or homogeneous nucleation of vapor bubbles may occur. For homogeneous nucleation, the vapor bubbles grow rapidly one towards the other, and when they touch each other the bubbles "explode". In this stage, the liquid around the bubbles is teared, and a spray with small and uniform droplets is formed. In the literature, it seems that the efficiency of the homogenous flash boiling process is very low. In this work, we analyse this process and analyse it for possible energy losses.
\end{abstract}

\section{Keywords}

Flash boiling atomization, Homogeneous nucleation and Spray Formation.

\section{Introduction}

Over the years, different methods have been developed in order to obtain suitable sprays for different applications. The more important characteristics of a spray include the drops diameter, droplet size distribution, spray shape, flow velocity and mass flux. Former studies [1]-[7] show that the flash boiling method is one of the most efficient methods to obtain a spray with very small drops and with a uniform distribution. These are very relevant for many applications such as combustion systems, for which higher combustion efficiency and low pollution are important. Today, flash boiling sprays are widely used to generate fine sprays in air refreshers, insect fighting, painting and some pharmaceutical applications. The flash boiling obtained by pressure reduction of compressed liquid bellow the saturation pressure.

The flash boiling spray is generated under well determined specific thermodynamic conditions. Based on the Levy et al. [8] model, the process is divided into three areas. When a liquid having a high vapor pressure, in the container (Fig. 1 area a), is discharged to a low pressure ambient through a orifice, ( $i$-e area). Under these conditions the rapid depressurization, results in a high bubbles nucleation, (point $n$ ). Vapor bubbles with radius $R_{c r}$ are created, and grow one towards another up to the point in which they touch each other (point $t$ ) and tear the liquid around them into small and relatively uniform droplets (area $d$ ).

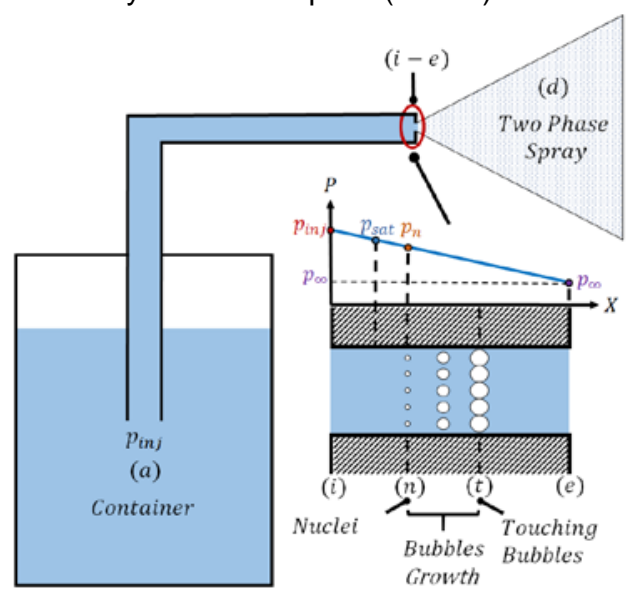

Figure 1. Homogenous flash boiling process sketch. 


\section{Bubbles nucleation}

Bubbles nucleation is one of the most important mechanisms in generation of the flash boiling spray. Depending on the thermodynamic conditions, two types of nucleation may occur. First, the bubbles generated on the orifice wall defined as a heterogeneous nucleation. Second, the bubbles nucleation occurs in the fluid bulk as known as homogeneous nucleation. Under homogeneous regime the bubbles nucleation rate is greater than the heterogeneous rate, and thus, the spray is finer [5], [7]. In addition, under this regime a simple orifice (Fig. 1) is required, therefore it may be applicable for fuel injection systems in engines and combustors.

The desirable homogenous nucleation occurrence depends on two criteria based on extensive experimental work. The Avedisian [9] criterion is on the initial temperature, i.e. the initial temperature is higher than $90 \%$ of the critical temperature, $T_{i n j}>0.9 T_{c r}$. In addition, Hutcherson et al. [10], [11] determined that the depressurization rate limit is $400 \mathrm{MPa} / \mathrm{s}$ for homogenous nucleation.

During the nucleation bubbles are generating in a various sizes. On each bubble two forces are acting. One, causing the bubble to increase, is the pressure difference across the bubble surface. Meaning, between inside bubble pressure, $p_{v}$, and liquid surrounding pressure, $p_{l}$. On the other hand, the bubble surface tension, $\sigma$, is acting to shrink it, when the surface tension is calculated by Sher et al. [12] method. The critical radius is defined by Young-Laplace equation, Eq. 1, and determines the collapsing or bubble spontaneously growing.

$$
R_{c r}=\frac{2 \sigma}{p_{v}-p_{l}}
$$

The nucleation flux density is very important quantity in flash boiling sprays. The homogeneous nucleation flux density suggestion, in seedy state system, depends on the critical size bubbles generation quantity and the bubble grows rate from the critical size. Thus, the expression for number of bubbles formed during a time unit per unit volume is [13].

$$
J=N_{A}^{\frac{3}{2}}\left(\frac{3 \rho_{l}^{2} \sigma}{\pi \bar{M}^{3}}\right)^{1 / 2} \exp \left[-\frac{16 \pi \sigma^{3}}{3 k_{B} T_{l}\left[\gamma p_{\text {sat }}\left(T_{l}\right)-p_{l}\right]^{2}}\right]
$$

where $T_{l}$ is the liquid initial temperature, $p_{s a t}\left(T_{l}\right)$ is the saturation pressure at initial temperature, $\gamma=\exp \left\{\left[p_{l}-p_{\text {sat }}\left(T_{l}\right)\right] / \rho_{l} \bar{R} T_{l}\right\}$ and the pressure difference is obtained by Redlich - Kwong equation of state (EOS).

\section{Bubbles growth}

When stable vapor bubble is formed, it spontaneously grow. The control growth type and the bubbles growth rate influence the spray droplets characteristics. This bubble growth process is very complicated. The momentum and mass conservation are coupled and non-linear. Furthermore, at the bubble wall, between the vapor and the liquid, there is hydrodynamic and thermal interaction. When, the Generalized Rayleigh-Plesset equation of motion describe a spherical vapor bubble growth in spherical coordinates, in infinite liquid pool.

$$
R \frac{d^{2} R}{d t^{2}}+\frac{3}{2}\left(\frac{d R}{d t}\right)^{2}=\frac{1}{\rho_{l}}\left(p_{v}-p_{\infty}-\frac{2 \sigma}{R}-\frac{4 \mu}{R} \frac{d R}{d t}\right)
$$

Where $R, \dot{R}$ and $\ddot{R}$ are radius, velocity and acceleration of the bubble radius growth respectively, and $p_{\infty}$ is the liquid pressure outside the boundary layer. Because it is not possible to solve this equation analytically without assumptions, the process of their growth can be divided into three stages:

1. Inertia control growth.

2. Coupled inertia and thermal-diffusion control growth.

3. Thermal-diffusion control growth.

When a bubble is formed, the temperature inside the bubble is assumed to be equal to the surrounding liquid temperature $\left(T_{v}=T_{\infty}\right)$, and the pressure inside the bubble is the saturation pressure at this temperature $\left(p_{v}=\right.$ $p_{\text {sat }}\left(T_{\infty}\right)$ ). During the first stage of the bubble growth, the limiting factor of the bubbles growth rate is the outward acceleration of the liquid around it. The growth rate of the bubble has been shown by Rayleigh [14] to be at the form of:

$$
R=A t
$$

In the final stage, the bubble becomes bigger, therefore, a larger amount of vapor is needed in order to increase its size. Furthermore, the pressure of the bubble decreases until it is equal to the pressure of the liquid surrounding $\left(p_{v}=p_{\infty}\right)$, and the bubble temperature is according to the saturation temperature at this pressure $\left(T_{v}=T_{s a t}\left(p_{\infty}\right)\right)$. As a result of the bubble growth, this layer of the surrounding liquid cools down and create a temperature difference. This temperature difference drives a thermal diffusion from the liquid into the bubble. Thus, at the third stage, the heat that can be supplied to the bubble walls is the limiting factor of the growth rate of the bubble. This stage of the bubble growth is shown by Plesset and Zwick [15] to be:

$$
R=B \sqrt{t}
$$


In the intermediate stage, the bubble grows and both the temperature and the pressure decreases. The limiting factor of the bubbles growth is the combination between the growth rate by inertia and the thermal diffusion. Mikic et al. [16] showed that the combined effects of the inertia and thermal diffusion determine the growth rate of the bubble:

$$
\begin{aligned}
& R^{*}=\frac{2}{3}\left[\left(t^{*}+1\right)^{\frac{3}{2}}-\left(t^{*}\right)^{\frac{3}{2}}-1\right] \\
& R^{*}=\frac{A}{B^{2}} R, \quad t^{*}=\left(\frac{A}{B}\right)^{2} t \\
& A=\left[\frac{2}{3} \frac{\left(T_{\infty}-T_{\text {sat }}\right) h_{f g} \rho_{v}}{T_{\text {sat }} \rho_{l}}\right]^{\frac{1}{2}}, \quad B=\left[\frac{12}{\pi} J a^{2} \alpha\right]^{\frac{1}{2}} \\
& J a=\frac{\rho_{l_{\text {inj }}} c_{p_{\text {inj }}}\left[T_{\text {inj }}-T_{\text {sat }}\left(p_{\infty}\right)\right]}{\rho_{v_{n}} h_{f g_{\text {inj }}}}
\end{aligned}
$$

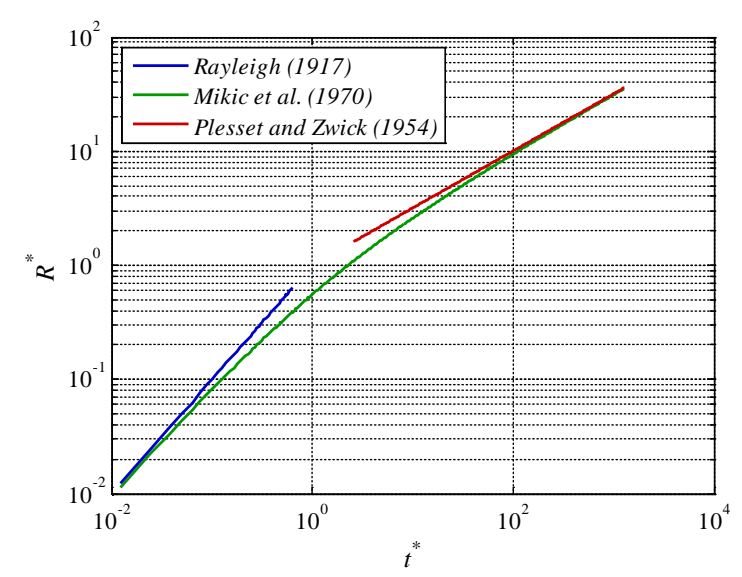

Figure 2. Mikic et al. [16], Plesset \& Zwick [15] and Rayleigh [14] models for predicting bubble growth in superheated R-22.

A better agreement to experiments of the bubble growth rate can be achieved by using numerical models. Robinson and Judd [17] offered a numerical model that can solve the coupling between the three following equations:

1. Generalized Rayleigh-Plesset equation, (Eq. 3).

2. Energy conservation equation of the bubble walls:

$$
h_{f g} \frac{\partial}{\partial t}\left(\frac{4}{3} \pi R^{3} \rho_{v}\right)=\frac{1}{4 \pi R^{2}} \int_{A_{s}} k_{l}\left(\frac{\partial T}{\partial r}\right)_{r=R} d A
$$

3. Energy equation outside the bubble:

$$
\frac{\partial T}{\partial t}+\frac{R^{2}}{r^{2}} \frac{d R}{d t} \frac{\partial T}{\partial r}=\alpha_{l}\left(\frac{\partial^{2} T}{\partial^{2} r}+\frac{2}{r} \frac{\partial T}{\partial r}\right)
$$

Eqs. (3), (7) and (8) are solved using the forth order Runga-Kutta numerical method.
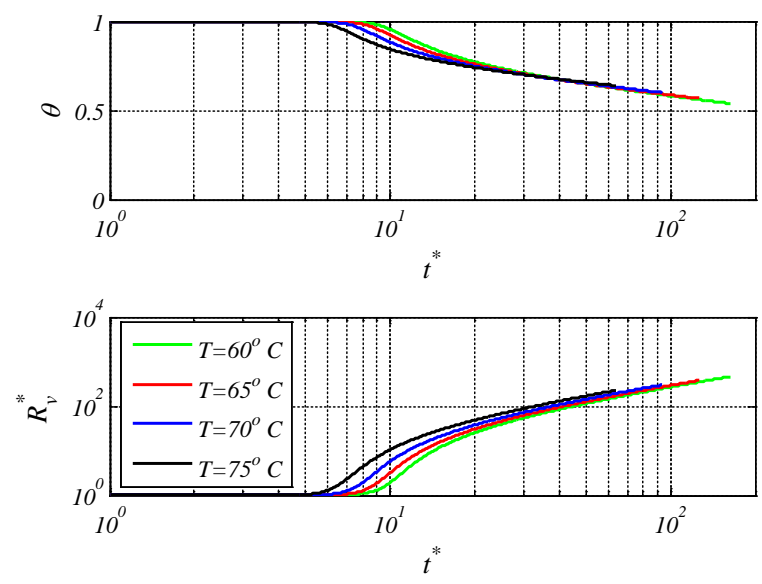

Figure 3. Dimensionless bubble radius and bubble vapor temperature versus dimensionless time, using Robinson and Judd [17] method for a few injection temperatures. 


\section{Energy mechanisms}

During the homogenous flash boiling generation spray process, the compressed liquid energy, in the container, is transformed into a several different energy mechanisms. By the energy partition model investigation, it is possible to examine the effect of the compressed liquid properties on the spray properties. This model is based on the flash boiling generation spray, the homogenous nucleation rate and Robinson and Judd [17] numerical bubble growth model, With the steady state steady flow (SSSF) assumption.

In the initial condition, when the compressed liquid is in the container (Fig. 1 area a). The liquid's energy will be its availability [18]:

$$
\Psi_{i n j}=\dot{m}_{l_{i}}\left[\left(h_{i n j}-h_{0}\right)-T_{0}\left(s_{i n j}-s_{0}\right)\right]
$$

where $h$ and $s$ are the enthalpy and the entropy respectively, the subscripts inj and 0 denotes to compressed liquid (container) and surrounding conditions. The mass flow rate in the orifice is:

$$
\dot{m}_{l}=\rho_{l_{i}} \bar{U}_{i} A_{N}=\dot{\phi}_{l_{i}} A_{N}
$$

Where $A_{N}$ is the orifice cross section area, $\dot{\phi}_{l}$ is the mass flux and $\bar{U}_{l_{i}}$ is the mean flow velocity, under the assumption that the flow isn't chocked.

When the liquid flow throw the orifice, rapid depressurization occurs and linearly pressure decrease is assumed. In consequence of the depressurization, a massive bubble nucleation occurs. To simplify, we assume that the formed bubbles are spherical and with uniform arrangement, Face- Centered Cubic (FCC) (Fig. 4) and stay that way for all of the growth process until they touch each other at point ( $t$ ) (Fig. 1). Furthermore, we assume that those kernels are formed in a uniform flow cross section, with width unit sell of (a) (Fig. 4). Also, we assume that the flow is adiabatic, meaning that there is no heat transfer from the liquid to the orifice walls and there is no relative flow between the bubbles and the liquid.

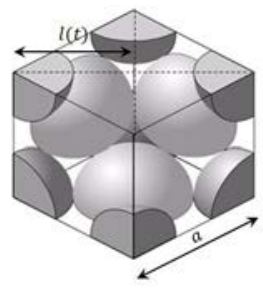

Figure 4. Face-Centred Cubic (FCC) unit cell.

According to those assumptions, the distance between the bubbles, $l$, is constant and can be evaluated the following way.

$$
l=\left(\frac{\bar{U}_{i}}{J}\right)^{1 / 4}
$$

During the process, from the moment the bubbles are formed $(n)$ until the moment they touch each other ( $t$ ) (areas $n$ and $t$ ), the energy can be expressed by using the following mechanisms:

- The flow kinetic energy flux of the orifice entrance:

$$
E_{k}^{\prime \prime}(t)=\dot{\phi}_{l_{i}} \frac{\bar{U}(t)^{2}}{2}
$$

- The evaporation energy flux of the liquid into the bubbles:

$$
E_{h_{n}}^{\prime \prime}=\frac{\dot{N}}{A_{N}}\left[\frac{4}{3} \pi R_{0}^{3} \rho_{v_{n}} h_{f g_{n}}+\int_{0}^{t} \dot{m}_{e v p}(t) h_{f g}(t) d t\right]
$$

- The mechanical work invested when the bubbles push the liquid while growing:

$$
E_{p}^{\prime \prime}(t)=4 \pi \frac{\dot{N}}{A_{N}} \int_{0}^{R(t)} p_{l}(t) r^{2} d r
$$

- The bubbles surface tension energy in the liquid a moment before the explosion:

$$
E_{\sigma}^{\prime \prime}(t)=\dot{N} 4 \pi \sigma R(t)
$$




\section{Spray characteristic}

After the bubbles touch each other, they explode and spread the liquid between them into a little droplets, so the spray is created (area $d$ ). Assuming that the number of bubbles is equal to the number of droplets, by mass conservation at the liquid phase, it is possible to evaluate the average radius of the formed droplets.

The efficiency of the process is defined by the following [1]:

$$
\eta=\frac{E_{\sigma_{d}}}{\Psi_{\text {inj }}-\Psi_{0}}
$$

When the droplets surface tension energy defined by:

$$
E_{\sigma_{d}}^{\prime \prime}=4 \pi \sigma_{d} \sum_{j=1}^{\dot{N}}\left(\frac{D_{j}}{2}\right)^{2}
$$

\section{Results and discussion}

At the following, we can see the dimensionless energy distribution of various mechanisms from the bubbles formation, point $(n)$ until they touch each other, point $(t)$. It is obtained based on the bubbles formation rate and the bubble growth rate model.

At the following figure, we can see the dimensionless energy distribution as function of the dimensionless time, for injection temperature $T_{i n j}=340 \mathrm{~K}$, meaning $93 \%$ of the critical temperature. A large part of the energy is converted into kinetic energy, to move the liquid throw the orifice as a result of the pressure differences.

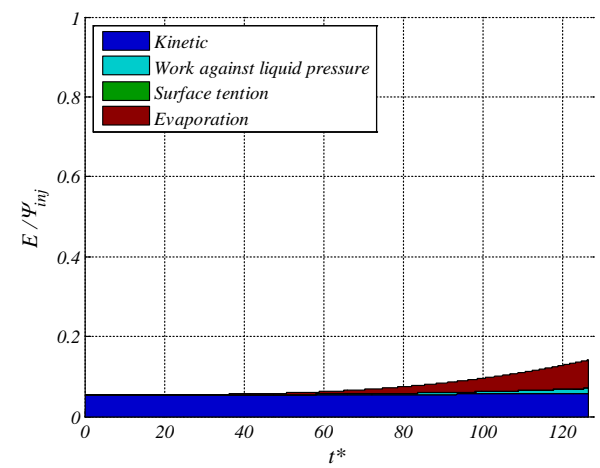

Figure 5. Dimensionless energy distribution as function of dimensionless time, from the bubbles formation, point $(n)$ until they touch each other, point $(t)$, for injection temperature $T_{i n j}=340 \mathrm{~K}$.

The following figures present the dimensionless energy distribution as function of the dimensionless temperature. In the left side (Fig 6.a) at the bubbles formation moment, point (n), and in the right side (Fig 6.b) the moment the bubbles touch each other, point $(t)$.

Most of the energy is converted into evaporation of the bubbles during their growth. Also, a significant amount of energy is wasted when the bubbles push the liquid around them. Furthermore, a small amount of energy is accumulated in the surface tension of the bubbles. Another interesting thing that can be concluded is that the energy of the different mechanisms increases with the liquid injection temperature.

(a)

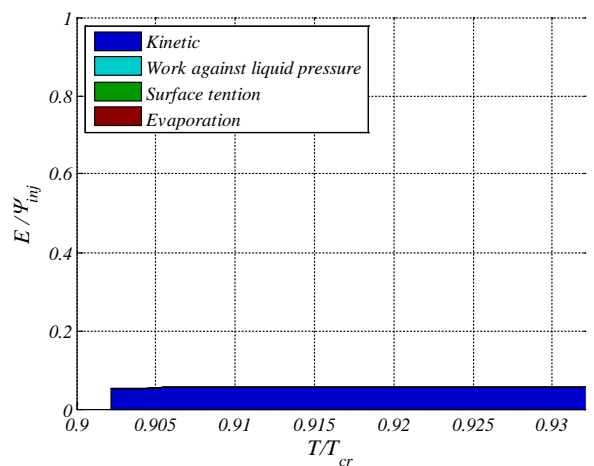

(b)

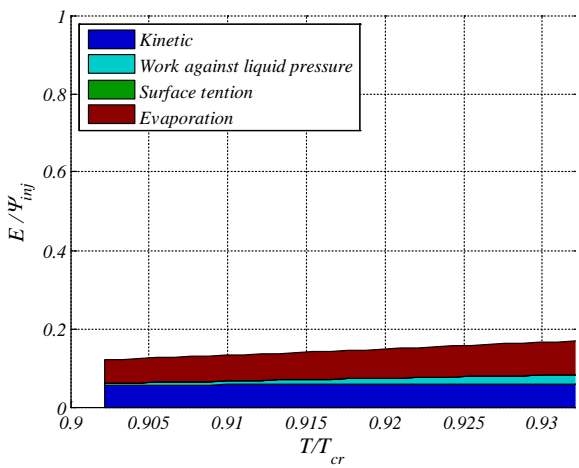

Figure 6. Dimensionless energy distribution as function of the dimensionless temperature. In (a), at the bubbles formation moment and in (b), at the moment the bubbles touch each other. 
Fig. 7.a shoes the average droplets diameter and Fig. 7.b presents the process efficiency as function of the dimensionless temperature for $\mathrm{CHCIF}_{2}$, Chlorodifluoromethane (R-22). It is possible to distinguish that the droplets radius reduces linearly when the temperature increases.

(a)

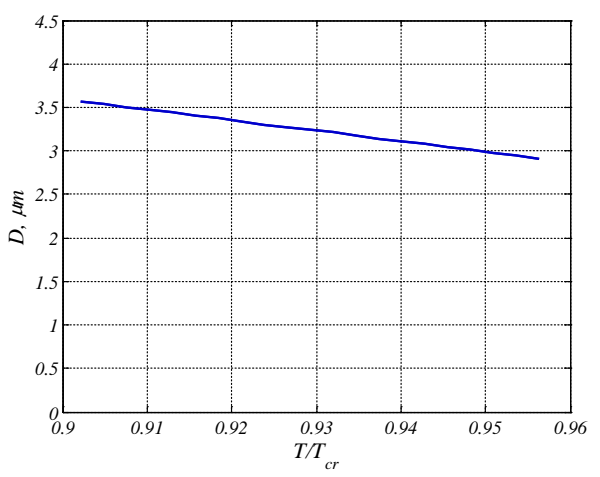

(b)

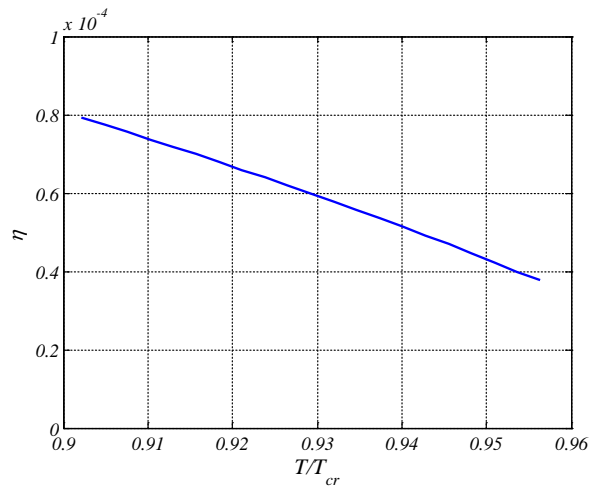

Figure 7. Average droplets diameter (in a) and process efficiency (in b) as function of the dimensionless temperature.

\section{Conclusions}

The current model present the energy distribution along the jet breaking process into a spray using homogenous flesh boiling. The model can evaluate changes of energy as a result of condition changes or the compressed liquid characteristics. Furthermore, the model can evaluate the sprays droplet radius. Using an experiment comparison allows to add a correction factor for the models assumptions. Homogeneous nucleation can be used in injection systems, such as combustors and engines because it can be achieved using a simple atomizer construction

\section{Nomenclature}

$A_{N} \quad$ Orifice cross section $\left[\mathrm{m}^{2}\right]$

$D \quad$ Diameter [m]

h Enthalpy $\left[\mathrm{Jg}^{-1}\right]$

nucleation rate $\left[\mathrm{n} \mathrm{m}^{-3} \mathrm{~s}^{-1}\right]$

Ja Jacobs number [- ]

$k \quad$ Thermal conduction $\left[W \mathrm{~m}^{-1} \mathrm{~K}^{-1}\right]$

$k_{b} \quad$ Boltzmann constant $\left[J K^{-1}\right]$

$l \quad$ Length between bubbles $[\mathrm{m}]$

$\dot{m} \quad$ Mass flow rate $\left[\mathrm{kg} \mathrm{s}^{-1}\right]$

$\bar{M} \quad$ Molar mass $\left[\mathrm{kg} \mathrm{mol}^{-1}\right]$

$N_{A} \quad$ Avogadro number [-]

$p \quad$ Pressure $[\mathrm{Pa}]$

$R \quad$ Bubble radius $[\mathrm{m}]$

$\bar{R} \quad$ Gas constant $\left[\mathrm{K}^{-1} \mathrm{~kg}^{-1}\right.$ ]

$s \quad$ Entropy $\left[\mathrm{Jg}^{-1} \mathrm{~K}^{-1}\right]$

$T$ Temperature $[K]$

$t \quad$ Time $[s]$

$\bar{U} \quad$ Average velocity $\left[m s^{-1}\right]$

$\theta \quad$ Dimensionless temperature [-]

$\mu \quad$ Dynamic viscosity $[\mathrm{Pas}]$

$\rho \quad$ Density $\left[\mathrm{kg} \mathrm{m}^{-3}\right]$

$\sigma \quad$ Surface tension $\left[\mathrm{N} \mathrm{m}^{-1}\right]$

$\dot{\phi} \quad$ mass flux $\left[\mathrm{kg} \mathrm{s}^{-1} \mathrm{~m}^{-2}\right]$

$\Psi \quad$ Availability $[W]$

$\begin{array}{ll}\text { Indices } & \\ \text { cr } & \text { Critic } \\ d & \text { Droplets } \\ h_{n} & \text { Evaporation energy } \\ \text { inj } & \text { Injection } \\ k & \text { Kinetic energy } \\ l & \text { Liquid } \\ n & \text { Nucleation } \\ p & \text { Mechanical work } \\ \text { sat } & \text { Saturation } \\ t & \text { Bubble exploding } \\ v & \text { Vapor } \\ & \\ 0 & \text { Surrounding conditions } \\ \infty & \text { Outside of the boundary layer } \\ * & \text { Dimensionless } \\ \sigma & \text { Surface tension energy }\end{array}$




\section{References}

[1] E. Sher and C. Elata, "Spray formation from pressure cans by flashing," Ind. Eng. Chem. Process Des. Dev., vol. 16, no. 2, pp. 237-242, 1977.

[2] T. Bar-Kohany and E. Sher, "Effervescent atomization under sub-sonic and choked conditions-a theoretical approach," Chem. Eng. Sci., vol. 59, no. 24, pp. 5987-5995, Dec. 2004.

[3] T. Bar-Kohany, I. Sher, and E. Sher, "Choked flow of a bubbly mixture through an effervescent and flashboiling atomizer: A theoretical approach," At. Sprays, vol. 17, no. 5, 2007.

[4] E. Sher, T. Bar-Kohany, and A. Rashkovan, "Flash-boiling atomization," Prog. Energy Combust. Sci., vol. 34, no. 4, pp. 417-439, Aug. 2008.

[5] M. Levy, Y. Levy, and E. Sher, "Spray structure as generated under homogeneous flash boiling nucleation regime," Appl. Therm. Eng., vol. 73, no. 1, pp. 416-423, Dec. 2014.

[6] T. Bar-Kohany and M. Levy, "STATE OF THE ART REVIEW OF FLASH-BOILING ATOMIZATION," At. Sprays, vol. 26, no. 12, 2016.

[7] M. Levy, Y. Levy, and E. Sher, "EFFECT OF THE PROPELLANT MASS FRACTION IN A BINARY MIXTURE ON THE SPRAY CHARACTERISTICS AS GENERATED BY HOMOGENEOUS FLASH BOILING," At. Sprays, vol. 26, no. 12, 2016.

[8] M. Levy, Y. Levy, and E. Sher, "Diameter Estimation of Droplets Under Homogeneous Flash Boiling," presented at the ILASS 2016, Brighton, England, 2016.

[9] C. T. Avedisian, "The Homogeneous Nucleation Limits of Liquids," J. Phys. Chem. Ref. Data, vol. 14, no. 3, pp. 695-729, 1985.

[10] M. N. Hutcherson, R. E. Henry, and D. E. Wollersheim, "Two-Phase Vessel Blowdown of an Initially Saturated Liquid-Part 1: Experimental,” J. Heat Transf., vol. 105, no. 4, pp. 687-693, 1983.

[11] M. Hutcherson, R. Henry, and D. Wollersheim, "Two-Phase Vessel Blowdown of an Initially Saturated Liquid-Part 2: Analytical," J. Heat Transf., vol. 105, no. 4, pp. 694-699, 1983.

[12] I. Sher, S. Haber, and G. Hetsroni, "A new state model of liquid-vapor interfaces-to yield analytical expression for surface tension," Chem. Eng. Sci., vol. 60, no. 3, pp. 711-716, Feb. 2005.

[13] V. P. Carey, "Liquid-vapor phase-change phenomena," 1992.

[14] Lord Rayleigh, "VIII. On the pressure developed in a liquid during the collapse of a spherical cavity," Lond. Edinb. Dublin Philos. Mag. J. Sci., vol. 34, no. 200, pp. 94-98, 1917.

[15] M. S. Plesset and S. A. Zwick, "The Growth of Vapor Bubbles in Superheated Liquids," J. Appl. Phys., vol. 25, no. 4, p. 493, 1954.

[16] B. Mikic, W. Rohsenow, and P. Griffith, "On bubble growth rates," Int. J. Heat Mass Transf., vol. 13, no. 4, pp. 657-666, 1970.

[17] A. J. Robinson and R. L. Judd, "The dynamics of spherical bubble growth," Int. J. Heat Mass Transf., vol. 47, no. 23, pp. 5101-5113, Nov. 2004.

[18] E. Sher and M. Zeigerson-Katz, "Spray formation by flashing of a binary mixture: An energy balance approach," At. Sprays, vol. 6, no. 4, 1996. 